\title{
sPLINK: a hybrid federated tool as a robust alternative to meta-analysis in genome-wide association studies
}

\author{
Reza Nasirigerdeh ${ }^{1,2^{*}} \mathbb{D}$, Reihaneh Torkzadehmahani ${ }^{1}$, Julian Matschinske ${ }^{3}$, Tobias Frisch ${ }^{4}$, Markus List ${ }^{5}$, \\ Julian Späth ${ }^{3}$, Stefan Weiss ${ }^{6}$, Uwe Völkerr ${ }^{6}$, Esa Pitkänen ${ }^{7,8}$, Dominik Heider ${ }^{9}$, Nina Kerstin Wenke ${ }^{3}$, \\ Georgios Kaissis 1,2,12,13, Daniel Rueckert ${ }^{1,2,12}$, Tim Kacprowski ${ }^{10,11 \dagger}$ and Jan Baumbach $3,4 \dagger$
}

*Correspondence:
reza.nasirigerdeh@tum.de
${ }^{\dagger}$ Tim Kacprowski and Jan
Baumbach are joint senior authors.
${ }^{1}$ Al in Medicine and Healthcare,
Technical University of Munich,
Munich, Germany
${ }^{2}$ Klinikum rechts der Isar, Munich,
Germany
Full list of author information is
available at the end of the article

The Author(s). 2022 Open Access This article is licensed under a Creative Commons Attribution 4.0 International License, which permits use, sharing, adaptation, distribution and reproduction in any medium or format, as long as you give appropriate credit to the original author(s) and the source, provide a link to the Creative Commons licence, and indicate if changes were made. The images or other third party material in this article are included in the article's Creative Commons licence, unless indicated otherwise in a credit line to the material. If material is not included in the article's Creative Commons licence and your intended use is not permitted by statutory regulation or exceeds the permitted use, you will need to obtain permission directly from the copyright holder. To view a copy of this licence, visit http://creativecommons.org/licenses/by/4.0/. The Creative Commons Public Domain Dedication waiver (http://creativecommons.org/publicdomain/zero/1.0/) applies to the data made

available in this article, unless otherwise stated in a credit line to the data.

\section{Background}

Genome-wide association studies (GWAS) test millions of single nucleotide polymorphisms (SNPs) to identify possible associations between a specific SNP and disease [1]. They have led to considerable achievements over the past decade including better comprehension of the genetic structure of complex diseases and the discovery of SNPs playing a role in many traits or disorders [2, 3]. GWAS sample size is an important factor in detecting associations, and larger sample sizes lead to identifying more associations and more accurate genetic predictors $[2,4]$.

PLINK [5] is a widely used open source software tool for GWAS. The major limitation of PLINK is that it can only perform association tests on local data. If multiple cohorts want to conduct collaborative GWAS to take advantage of larger sample sizes, they can pool their data for a joint analysis (Fig. 1a); however, this is close to impossible due to privacy 


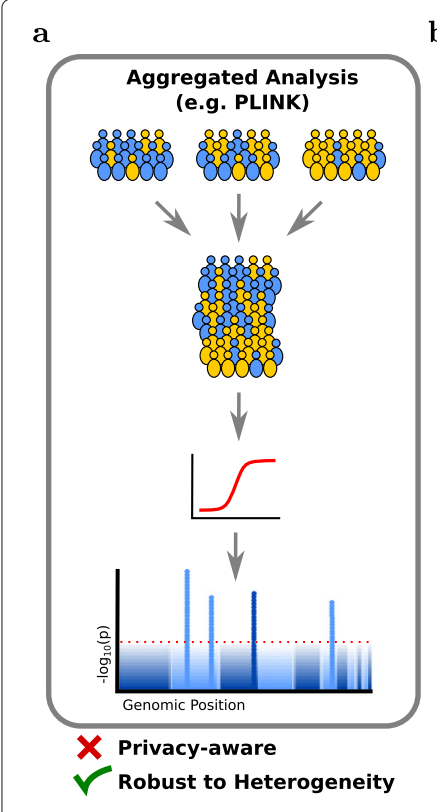

Aggregated analysis

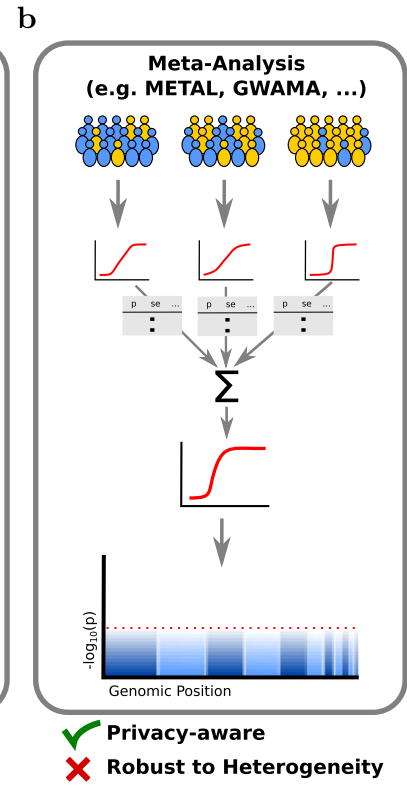

Meta-analysis

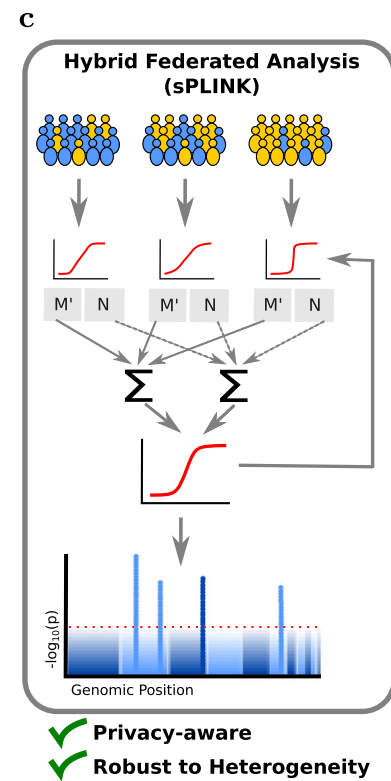

SPLINK

Fig. 1 Comparison of sPLINK (c), aggregated analysis (a), and meta-analysis (b) approaches: Aggregated analysis requires cohorts to pool their private data for a joint analysis. The meta-analysis approaches aggregate the summary statistics from the cohorts to estimate the combined p-values. In sPLINK, the cohorts calculate the model parameters $(M)$ from the local data and global model, generate noise $(N)$, and make the parameters noisy $\left(M^{\prime}\right)$ in an iterative manner. The aggregated noise and noisy parameters are in turn aggregated to update the global model or build the final model. sPLINK combines the advantages of the aggregated analysis and meta-analysis, i.e. robustness against heterogeneous data and enhancing the privacy of cohorts' data. Yellow/blue color indicates case/control samples

restrictions and data protection issues, especially concerning genetic and medical data. Hence, the field has established methods for meta-analysis of individual studies, where only the results and summary statistics of the individual analyses have to be exchanged [6] (Fig. 1b).

There are several software packages such as METAL [7], GWAMA [8], and PLINK [5] that implement different meta-analysis models including fixed or random effect models [9]. Although meta-analysis approaches are privacy-aware, i.e. the raw data is not shared with third parities, they suffer from two main constraints: first, they rely on detailed planning and agreement of cohorts on various study parameters such as meta-analysis model (e.g. fixed effect or random effect), meta-analysis tool (e.g., METAL or GWAMA), heterogeneity metric (e.g. Cochran's $Q$ or the $I^{2}$ statistic), the covariates to be considered, etc [4]. Second and more importantly, the statistical power of meta-analysis can be adversely affected in the presence of cross-study heterogeneity, leading to inaccurate estimation of the joint results and yielding misleading conclusions [10,11].

To address the aforementioned shortcomings, privacy-aware collaborative GWAS can be developed using homomorphic encryption (HE) [12], secure multi-party computation (SMPC) [13], and federated learning [14, 15]. In HE, the cohorts encrypt their private data and share it with a single server, which performs operations on the encrypted data from the cohorts to compute the association test results. In SMPC, there are several computing parties and the cohorts extract a separate secret share (anonymized chunk) [16] from 
the private data and send it to a computing party. The computing parties calculate intermediate results from the secret shares and exchange the intermediate results with each other. Each computing party computes the final results given all intermediate results. In federated learning, the cohorts extract model parameters (e.g. Hessian matrices) from the private data and share the parameters with a central server. The server aggregates the parameters from all cohorts to calculate the association test results.

Kamm et al. [17] and Cho et al. [18] proposed GWAS frameworks based on SMPC. The former developed simple association tests including Cochran-Armitage and chi-square $\left(\chi^{2}\right)$ and the latter implemented only the Cochran-Armitage test for trend. Shi et al. [19] presented an SMPC-based logistic regression framework for GWAS. Constable et al. [20] implemented an SMPC-based framework for minor allele frequency and chi-square computation. These frameworks inherit the limitations of SMPC itself: They follow the paradigm of "move data to computation," where they put the processing burden on a few computing parties. Consequently, they are computationally expensive [21] and are not scalable for large-scale GWAS. Moreover, they suffer from the colluding-parties problem [17] in which, if the parties send the secret shares of the cohorts to each other, the whole private data of the cohorts is exposed.

$\mathrm{Lu}$ et al. [22], Morshed et al. [23], and Kim et al. [24] developed chi-square, linear regression, and logistic regression tests using HE for GWAS, respectively. Sadat et al. [25] introduced the SAFETY framework based on HE and Intel Software Guard Extensions technology, which implements the linkage disequilibrium, Fisher's exact test, CochranArmitage test for trend, and Hardy-Weinberg equilibrium statistical tests. Similar to SMPC-based methods, they are not computationally efficient because a single server carries out operations over encrypted data, causing considerable overhead [26]. Additionally, HE-based methods introduce accuracy loss in the association test results [23, 24]. This is because HE only supports addition and multiplication, and as a result, non-linear operations in regression tests should be approximated using those two operations.

To address the computational limitation of HE/SMPC-based methods, the association tests can be implemented in a federated fashion. Federated learning-based methods follow the paradigm of "move computation to data," distributing the heavy computations among the cohorts while performing lightweight aggregation (simple operations such as addition and multiplication of the parameters) at the central server. Wang et al. [27] introduced EXPLORER for distributed logistic regression algorithm. EXPLORER is a model but not a tool for GWAS. Moreover, it does not provide a "guarantee for optimal global solution," implying that its results can be different from the aggregated analysis in general. GLORE $[28,29]$ implemented a federated logistic regression test but the parameter values computed by each cohort are revealed to the server.

Several hybrid federated frameworks including HyFed [30] have been introduced to improve the privacy of federated learning by hiding the local parameters of a cohort from third parties. HyFed is a suitable framework for developing federated GWAS algorithms because it provides enhanced privacy while preserving the accuracy of the results. It also supports federated mode, where different components can run in separate physical machines and securely communicate with each other over the Internet.

In this paper, we present a hybrid federated tool called sPLINK (safe PLINK) based on the HyFed framework for privacy-aware GWAS. sPLINK consists of four main components (Fig. 2): Web application (WebApp) to configure the parameters (e.g. association 


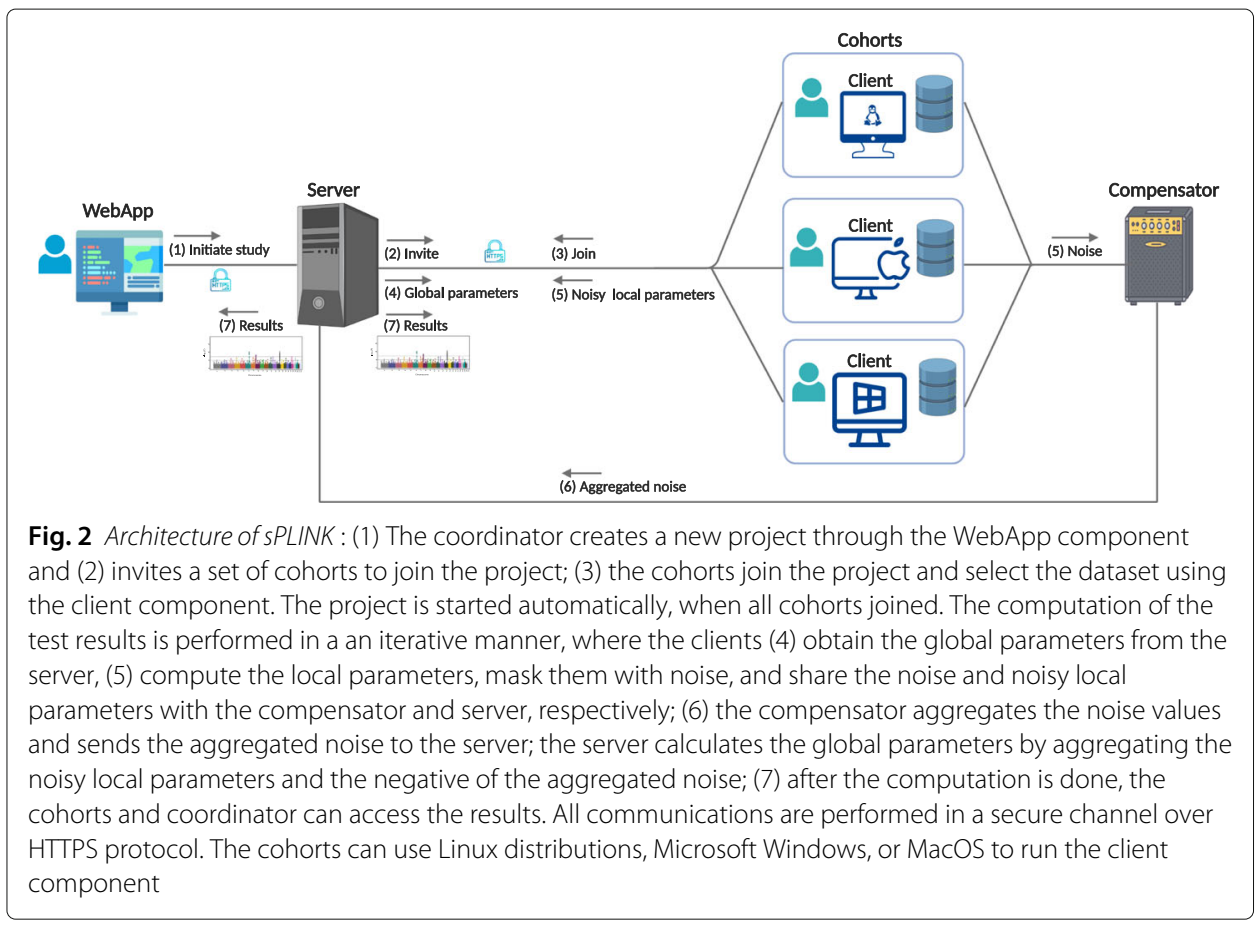

test) of the new study; client to compute the local parameters, mask them with noise, and share the noise with compensator and noisy local parameters with server; compensator to aggregate the noise values of the clients and send the aggregated noise to the server; server to compute the global parameters by adding up the noisy local parameters and the negative of the aggregated noise. Notice that the utility of the global model is preserved because the aggregated noise from the compensator cancels out the accumulated noise from the noisy local parameters during the aggregation.

Unlike PLINK, sPLINK is applicable to distributed data in a privacy-aware fashion. In $S P L I N K$, neither the private data of cohorts leaves the site nor the original values of the local parameters are revealed to the other parties (Fig. 1c). Contrary to the existing HE/SMPC-based methods, sPLINK is computationally efficient because heavy computations are distributed across the cohorts while simple aggregation is performed on the server and compensator. Compared to the current federated tools like GLORE, sPLINK not only provides enhanced privacy but also supports multiple association tests including logistic and linear regression [31], and chi-square [32] for GWAS.

The advantage of $S P L I N K$ over the meta-analysis approaches is twofold: usability and robustness against heterogeneity. sPLINK is easier to use for collaborative GWAS compared to meta-analysis. In $S P L I N K$, a coordinator initiates a collaborative study and invites the cohorts. The only decision the cohorts make is whether or not to join the study. After accepting the invitation, the cohorts just select the dataset they want to employ in the study. More importantly, sPLINK is robust to data heterogeneity (phenotype and confounding factors). It gives the same results as aggregated analysis even if the phenotype distribution is imbalanced or if confounding factors are distributed heterogeneously across cohorts. In contrast, meta-analysis tools typically lose statistical power in such imbalanced or heterogeneous scenarios (details in the "Results" section). 


\section{Results}

We first verify sPLINK by comparing its results with those from aggregated analysis conducted with PLINK for all three association tests on a real GWAS dataset from the SHIP study [33]. We refer to this dataset as the SHIP dataset, which comprises the records of 3699 individuals with serum lipase activity as phenotype. The quantitative version represents the square root transformed serum lipase activity, while the dichotomous (binary) version indicates if the serum lipase activity of an individual is above or below the 75th percentile. The SHIP dataset contains around 5 million SNPs as well as sex, age, smoking status (current-, ex-, or non-smoker), and daily alcohol consumption (in g/day) as confounding factors (Table 1).

We employ the binary phenotype for logistic regression and the chi-square test, and the quantitative phenotype for linear regression. We incorporate all four confounding factors in the regression models and no confounding factor in the chi-square test. We horizontally (sample-wise) split the dataset into four parts, simulating four different cohorts (Additional file 1: Table S1). PLINK computes the statistics for each association test using the whole dataset while $S P L I N K$ does it in a federated manner using the splits of the individual cohorts. To be consistent with PLINK, sPLINK calculates the same statistics as PLINK for the association tests.

We compute the difference between the $p$-values as well as the Pearson correlation coefficient $(\rho)$ of $p$-values from $S$ PLINK and PLINK. We use $-\log _{10}$ ( $p$-value) because the $p$-values are typically small and - $\log _{10}(p$-value) can be a better indicator of small $p$-value differences. According to Fig. $3 \mathrm{a}-\mathrm{c}$, the $p$-value difference is zero for most of the SNPs. We also observe that the maximum difference is 0.162 for a SNP in the linear regression. SPLINK and PLINK report $4.441 \times 10^{-16}$ and $3.058 \times 10^{-16}$ as $p$-values for the SNP, respectively. This negligible difference can be attributed to inconsistencies in floating point precision.

The correlation coefficient of $p$-values from SPLINK and PLINK for all three tests is $0 . \overline{99}$, which is consistent with the results of $p$-value difference from Fig. 3a-c. We investigate the overlap of significantly associated SNPs between SPLINK and PLINK. We

Table 1 Description of datasets

\begin{tabular}{|c|c|c|c|c|}
\hline Dataset & \# Samples & \# SNPs & Adjustments & Phenotype \\
\hline \multirow[t]{2}{*}{ SHIPa } & 3699 & $\sim 5 \mathrm{M}$ & $\begin{array}{l}\text { Sex, age, smoking status, } \\
\text { daily alcohol consumption }\end{array}$ & $\begin{array}{l}\text { SLA }{ }^{b} \text {, dichotomous ( } 75 \text { th } \\
\text { percentile, } 934 \text { cases, } 2765 \\
\text { controls) }\end{array}$ \\
\hline & & & & $\begin{array}{ll}\text { SLA, } & \text { quantitative, } \\
\text { Mean } \pm \mathrm{SD}^{\mathrm{C}} & 1.23 \pm 0.3\end{array}$ \\
\hline \multirow[t]{2}{*}{ COPDGene $^{d}$} & 5343 & $\sim 600 \mathrm{~K}$ & $\begin{array}{l}\text { Sex, age, smoking status, } \\
\text { pack years of smoking }\end{array}$ & $\begin{array}{l}\text { COPDe, dichotomous, } \\
\text { (2811 cases, } 2532 \text { controls) }\end{array}$ \\
\hline & & & & $\begin{array}{l}\mathrm{FEV}^{\mathrm{f}}{ }^{\mathrm{f}} \quad \text { quantitative, } \\
\text { Mean } \pm \mathrm{SD} 2.993 \pm 0.635\end{array}$ \\
\hline FinnGen & 135,615 & $\sim 1 \mathrm{M}$ & Sex and age & $\begin{array}{l}\text { Hypertension, dichoto- } \\
\text { mous, (34,257 cases, } \\
101,358 \text { controls) }\end{array}$ \\
\hline
\end{tabular}

astudy of Health in Pomerania

berum lipase activity

'Standard deviation

${ }^{d}$ Genetic Epidemiology of chronic obstructive pulmonary disease

e Chronic obstructive pulmonary disease

${ }^{f}$ Forced expiratory volume in one second 


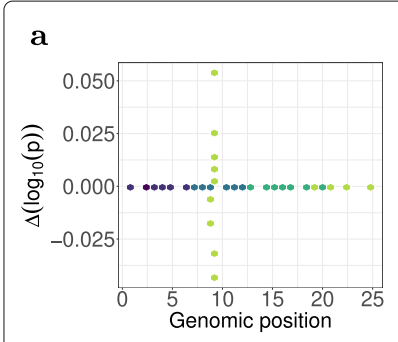

d

Logistic regression

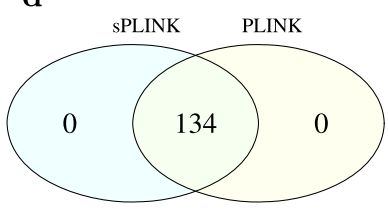

Logistic regression

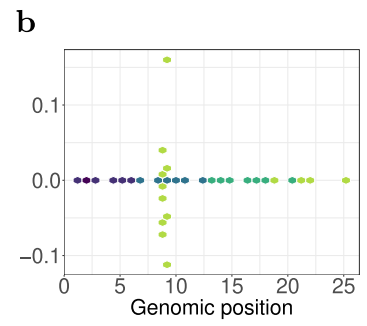

Linear regression$$
\text { e }
$$

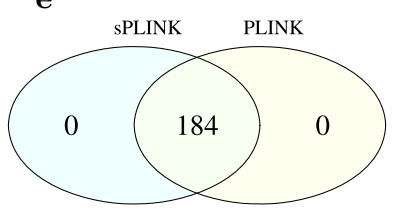

Linear regression
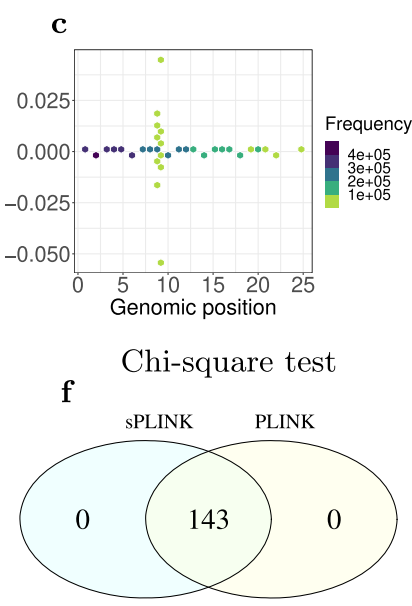

Chi-square test

Fig. $3 \Delta \log _{10}(p$-value) between sPLINK and PLINK as well as the set of SNPs identified by sPLINK and PLINK as significant for logistic regression $(\mathbf{a}, \mathbf{d})$, linear regression $(\mathbf{b}, \mathbf{e})$, and chi-square test $(\mathbf{c}, \mathbf{f})$, respectively. For most of the SNPs, the difference is zero, indicating that sPLINK gives the same $p$-values as PLINK. The negligible difference between $p$-values for the other SNPs can be attributed to differences in floating point precision. The spikes in some genomic positions are due to the strong association of the corresponding SNPs, which result in higher absolute error. SPLINK and PLINK also recognize the same set of SNPS as significant. Genomic positions (ticks in $\mathbf{a}-\mathbf{c}$ ) indicate chromosome numbers. The details of the experiments are available in Additional file 1: Table S1

consider a SNP as significant if its $p$-value is less than $5 \times 10^{-8}$ (genome-wide significance). PLINK and SPLINK recognize the same set of SNPs as significant (Fig. $3 \mathrm{~d}-\mathrm{f}$ ). Notably, the identified SNPs, e.g. rs8176693 and rs632111, lying in genes ABO (intronic) and FUT2 (3-UTR), respectively, have also been implicated in a previous analysis of this dataset [34]. We also leverage the Bonferroni significance threshold (which is $\approx 1 \times 10^{-8}$ for our tests) to compare the overlapping significant SNPs from SPLINK and PLINK. The results remain similar and the associated plot is available at Additional file 1: Fig. S1. These results indicate that $p$-values computed by $S P L I N K$ in a federated manner are the same as those calculated by PLINK on the aggregated data (ignoring negligible floating point precision error). In other words, the federated computation in $S P L I N K$ preserves the accuracy of the results of the association tests.

Next, we compare $S P L I N K$ with some existing meta-analysis tools, namely PLINK, $M E T A L$, and GWAMA. We leverage the COPDGene (non-hispanic white ethnic group) [35] and FinnGen (data release 3) [36] datasets. The COPDGene dataset has an equal distribution of case and control samples unlike the SHIP dataset. It contains 5343 samples (ignoring 1327 samples with missing phenotype value) and around 600K SNPs. We utilize chronic obstructive pulmonary disease (COPD) as the binary phenotype and include sex, age, smoking status, and pack years of smoking as confounding factors [37]. FinnGen is much larger dataset (in terms of sample size) compared to the SHIP and COPDGene datasets. It consists of 135,615 samples (ignoring 23 samples with missing phenotype value) and about 1 million SNPs. We use Hypertension as the (binary) phenotype and adjust for sex and age as confounding factors (Table 1).

To simulate cross-study heterogeneity [38] on the COPDGene dataset, we consider six different scenarios: Scenario I (Balanced), Scenario II (Slightly Imbalanced), Scenario III 
(Moderately Imbalanced), Scenario IV (Highly Imbalanced), Scenario V (Severely Imbalanced), and Scenario VI (Heterogeneous Confounding Factor) (Figs. 4a and 5). In each scenario, we partition the dataset into three splits with the same sample size (more details in Additional file 1: Table S2). The distribution of all four confounding factors is homogeneous (similar) across the splits for the first five scenarios. The splits have the same (and balanced) case-control ratio in Scenario I and Scenario VI but their case-control ratio is different for the imbalanced scenarios (Fig. 4a). In Scenario VI, the values of two confounding factors (i.e. smoking status and age) are homogeneously distributed among the splits; however, the distribution of sex and pack years of smoking is slightly and highly heterogeneous across the splits, respectively (Fig. 5). We obtain the summary statistics (e.g. minor allele, odds ratio, and standard error) for each split to conduct meta-analyses. The results are then compared to the federated analysis employing sPLINK. Figure $6 \mathrm{a}$ shows the Pearson correlation coefficient of $-\log _{10}$ ( $p$-value) between each tool and the aggregated analysis for all six scenarios. Figure 6c depicts the number of SNPs correctly identified as significant by the tools (true positives).

According to Fig. 6a, the correlation of $p$-values between $S P L I N K$ and the aggregated analysis is $\sim 1.0$ for all six scenarios, implying that sPLINK gives the same $p$-values as the aggregated analysis regardless of how phenotypes or confounding factors have been distributed across the cohorts. In contrast, the correlation coefficient for the meta-analysis tools shrinks with increasing imbalance/heterogeneity, indicating loss of accuracy. Figure $6 \mathrm{c}$ illustrates that sPLINK correctly identifies all four significant SNPs in all scenarios. In the balanced scenario, almost all meta-analysis tools perform well and recognize all significant SNPs. An exception is METAL, which misses one of them. However, they miss more and more significant SNPs as the phenotype imbalance across the splits increases. In the Highly Imbalanced and Severely Imbalanced scenarios, the meta-analysis tools cannot recognize any significant SNP. This is also the case if the distribution of some confounding factors becomes heterogeneous across the cohorts (Scenario VI). We checked the number of SNPs wrongly identified as significant by the tools (false positives) too. sPLINK has no false positive in any of the scenarios and the meta-analysis tools introduce zero or one false positive depending on the scenario.

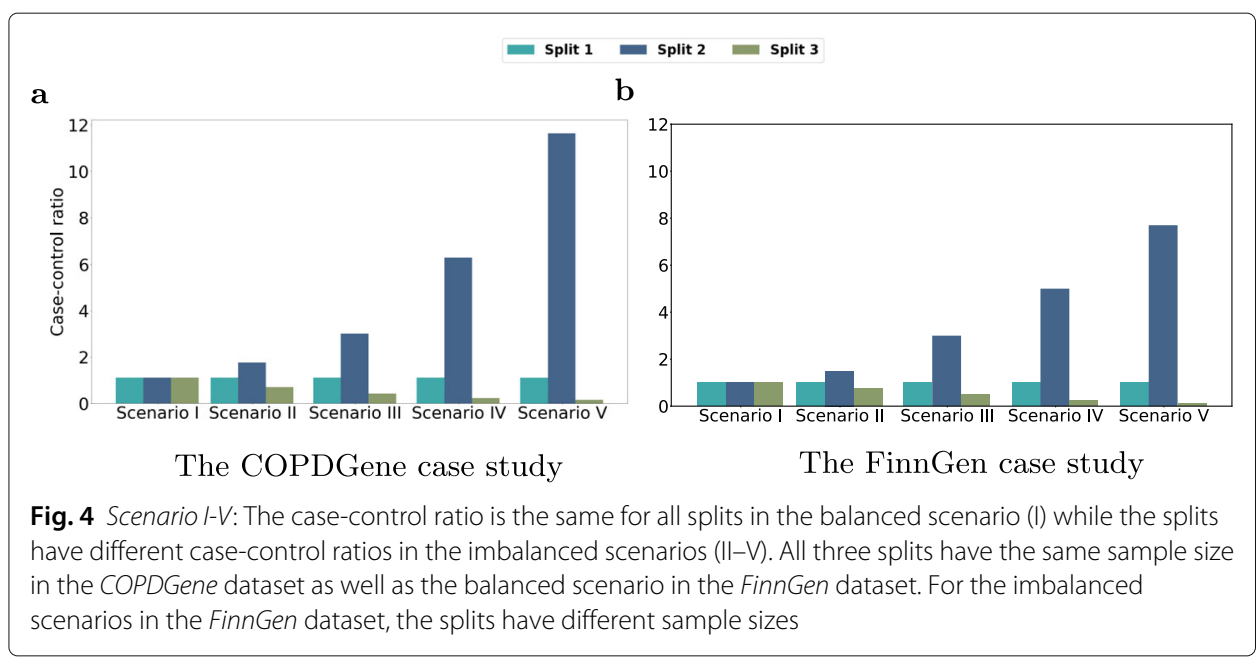




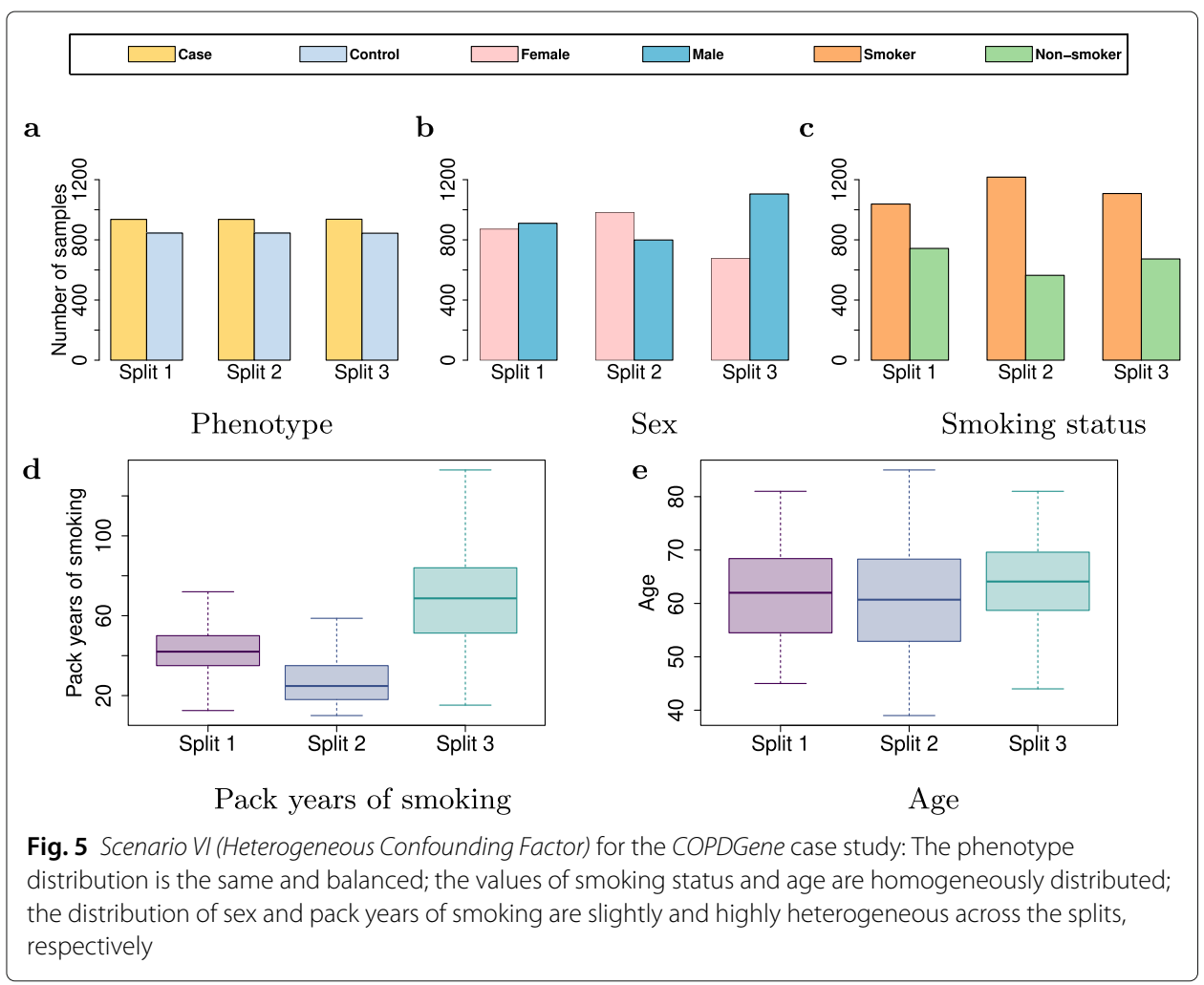

To show that our findings on the COPDGene dataset also hold true for a much larger dataset, we repeat the simulations on the FinnGen dataset (more details in Additional file 1: Table S3). Similar to the COPDGene case study, we divide the dataset into three splits and define Scenario I to Scenario V, where the splits have the same case-control ratio $(1.0)$ and sample size $(22,838)$ as in Scenario $I$ but different case-control ratios in the remaining scenarios (Fig. 4b); Unlike the COPDGene case study in which the sample size of the splits are equal for all scenarios including the imbalanced ones, the splits have different number of samples in the imbalanced scenarios of the FinnGen case study. For instance, split1, split2 and split3 have 22,838, 12,561, and 99,345 samples in Scenario V, respectively (a split with lower case-control ratio has larger sample size). It implies that the aggregated datasets have different number of samples in the scenarios, and as a result, there are different set of significant SNPs in each scenario of the FinnGen case study (total of 110, 116, 199, 304, and 446 significant SNPs in Scenario I to Scenario V, respectively).

Figures $6 \mathrm{~b}$ and $6 \mathrm{~d}$ illustrate the Pearson correlation coefficient and percentage of correctly identified significant SNPs for each scenario on the FinnGen case study, respectively. According to Fig. 6b, the correlation coefficient diminishes for the meta-analysis tools as the scenario becomes more and more imbalanced. This is also the case for the percentage of the SNPs correctly identified as significant by each meta-analysis tool (Fig. 6d). These results are consistent with those from the COPDGene case study. Moreover, we observed that the meta-analysis tools report high number of false positives (14-88) in Scenario $I V$. Thus, the limitations of meta-analysis tools towards class imbalance observed in the COPDGene dataset can be reproduced on a large dataset. However, sPLINK always provides the same results as PLINK with the aggregated analysis (the "Methods" section,

Figs. 3 and 6a, c). 


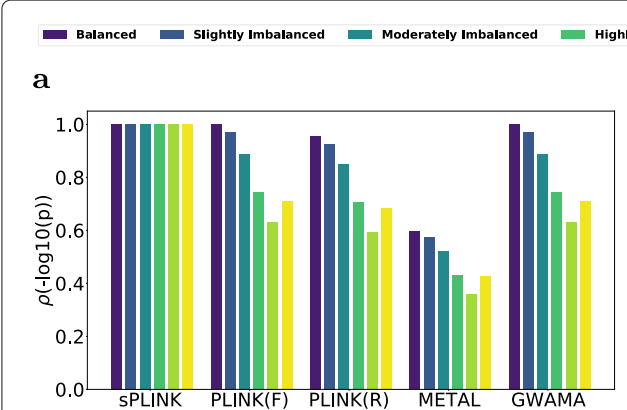

c

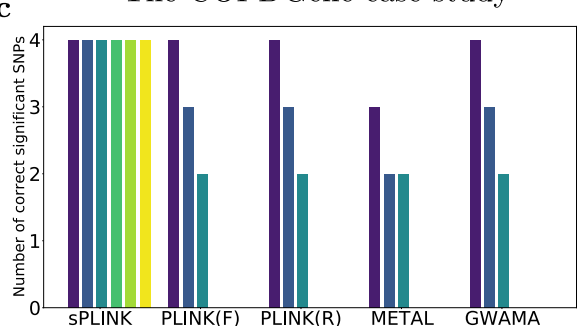

The COPDGene case study b

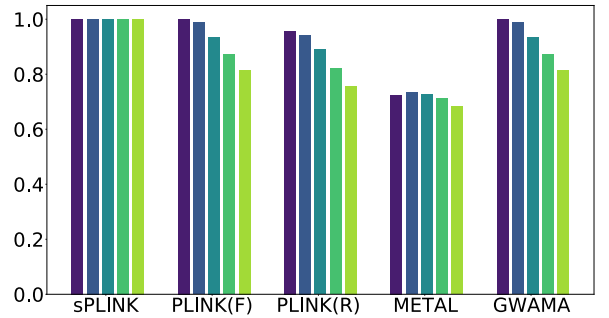

d

The FinnGen case study

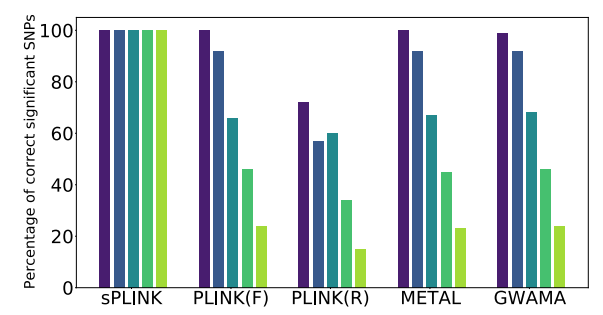

The FinnGen case study

Fig. 6 The Pearson correlation coefficient ( $\rho$ ) of - $\log _{10}(p$-value) between each tool and aggregated analysis $(\mathbf{a}, \mathbf{b})$ and the number (c) and the percentage (d) of SNPs correctly identified as significant (true positives) by each tool. $F$ and $R$ stand for fixed-effect and random-effect, respectively. The details of the experiments are available in Additional file 1: Table S2, and Table S3

We also leverage the Spearman correlation to check whether or not the meta-analysis tools maintain the ordering of significance compared to the aggregated analysis. Our results show that this is not the case, and the Spearman correlation values for the metaanalysis tools reduce as the phenotype imbalance across the splits increases, similar to the results from Fig. 6, where the Pearson correlation is used. The corresponding plot can be found in Additional file 1: Figure S2.

Table 2 shows a concise comparison between sPLINK and the state-of-the-art approaches. Unlike PLINK, SPLINK is privacy-aware, where the private data never leaves the cohorts. SPLINK is also robust against the imbalance/heterogeneity of phenotype/confounding factor distributions across the cohorts. SPLINK always delivers the same $p$-values as aggregated analysis and correctly identifies all significant SNPs independent of the phenotype or confounding factor distribution in the cohorts. In contrast, meta-analysis tools lose their statistical power in imbalanced phenotype scenarios, missing some or all significant SNPs. This is also the case if the phenotype distribution is balanced but the values of confounding factor(s) have heterogeneously been distributed across the datasets. Compared to the existing SMPC/HE-based approaches, sPLINK is computationally efficient and supports multiple association tests including chisquare and linear/logistic regression. SPLINK provides enhanced privacy by hiding the model parameters of each cohort from the third parties while federated learning-based frameworks such as GLORE reveal them to the server.

Finally, we measure the runtime and network bandwidth usage of sPLINK for each association test using the COPDGene dataset partitioned into three splits of the same sample 
Table 2 Comparison between sPLINK and the state-of-the-art approaches

\begin{tabular}{llllll}
\hline Tool/Study & $\begin{array}{l}\text { Privacy- } \\
\text { aware }\end{array}$ & $\begin{array}{l}\text { Robust to het- } \\
\text { erogeneity }\end{array}$ & $\begin{array}{l}\text { Computationally } \\
\text { efficient }\end{array}$ & $\begin{array}{l}\text { Linear } \\
\text { regres- } \\
\text { sion }\end{array}$ & $\begin{array}{l}\text { Logistic regres- } \\
\text { sion }\end{array}$ \\
\hline PLINK & $x$ & $\checkmark$ & $\checkmark$ & $\checkmark$ & $\checkmark$ \\
Meta-analysis & $\checkmark$ & $x$ & $\checkmark$ & $\checkmark$ & $\checkmark$ \\
Kamm et al. [17] & $\checkmark$ & $\checkmark$ & $x$ & $*$ & $x$ \\
Cho et al. [18] & $\checkmark$ & $\checkmark$ & $x$ & $*$ & $x$ \\
Morshed et al. [23] & $\checkmark$ & $x$ & $x$ & $\checkmark$ & $x$ \\
Kim et al. [24] & $\checkmark$ & $x$ & $x$ & $x$ & $\checkmark$ \\
GLORE [28] & $\checkmark$ & $\checkmark$ & $\checkmark$ & $x$ & $\checkmark$ \\
sPLINK & $\checkmark$ & $\checkmark$ & $\checkmark$ & $\checkmark$ & $\checkmark$
\end{tabular}

*The study supports the Cochran-Armitage test, which is computationally comparable to linear regression

size. We use COPD in chi-square as well as logistic regression and FEV1 in linear regression as phenotype. We include age, sex, smoking status, and pack years of smoking as confounding factors only for the regression tests. The server and WebApp packages are installed on a physical machine located at Freising (Germany) while the compensator is running on a machine at Odense (Denmark). Three commodity laptops located at Munich or Freising are running the client package and host the splits. They communicate with the server and compensator through the Internet. The system specification of the machines and laptops as well as the details of the experiments can be found in Additional file 1: Table S4 and S5.

Figure 7a plots the sPLINK's runtime for each association test. sPLINK computes the results for chi-square, linear regression, and logistic regression in $8 \mathrm{~min}, 20 \mathrm{~min}$, and $75 \mathrm{~min}$, respectively. Sending parameters from the clients to the server and compensator contributes the most in sPLINK's runtime. Compared to Kamm et al. [17], sPLINK is almost 13 times faster for chi-square test ( $8 \mathrm{~min}$ vs. $110 \mathrm{~min}^{1}$ ) with less powerful hardware, larger sample size (5343 vs. 1080), and more number of SNPs ( $\sim 580 \mathrm{~K}$ vs. $\sim 263 \mathrm{~K})$.

Figure $7 \mathrm{~b}$ depicts the network usage of $s$ PLINK. The clients, server, and compensator exchange total of $0.967 \mathrm{~GB}, 2.49 \mathrm{~GB}$, and 11.06 GB traffic in chi-square, linear regression, and logistic regression, respectively. Logistic regression has higher volume of traffic

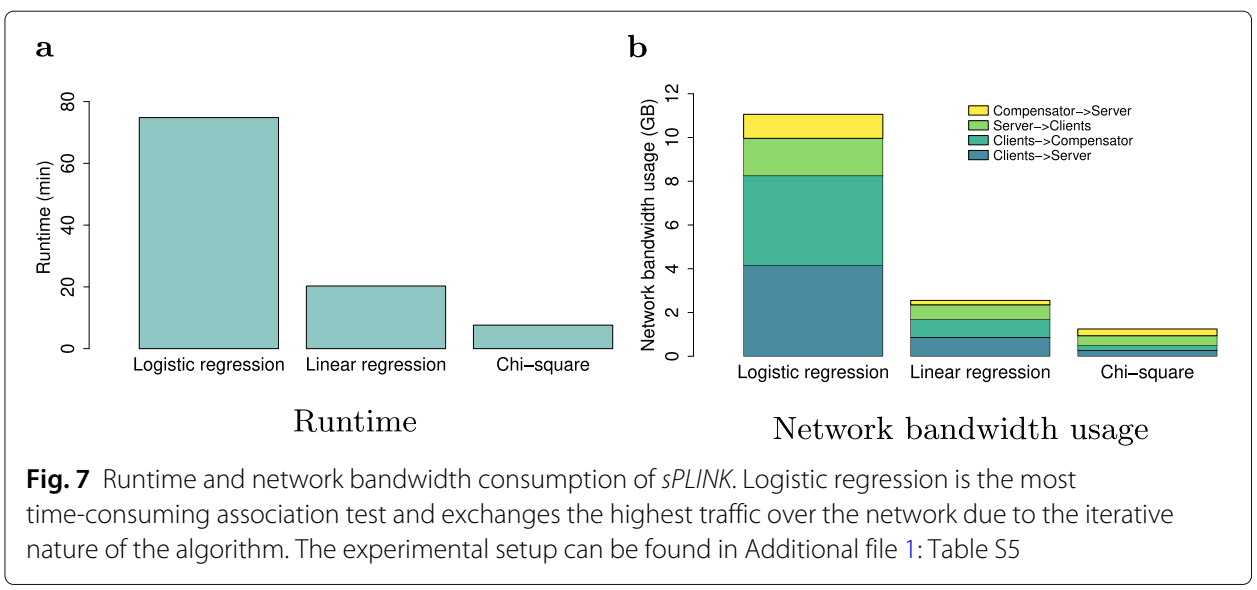

\footnotetext{
${ }^{1}$ The best result from Kamm et al. [17] has been considered.
} 
exchange because the computation of beta coefficients are performed in an iterative fashion. A fair comparison between SPLINK and SMPC-based frameworks from the network communication aspect is tricky. However, in general, (hybrid) federated learning-based approaches consume more network bandwidth than SMPC-based ones.

We also conduct a set of experiments to investigate how the runtime and network bandwidth consumption of $s$ PLINK change with varying number of samples, SNPs, and clients. The results demonstrate that the traffic exchanged over the network is independent of the sample size and linearly increases with the number of SNPs and clients (as expected). Moreover, runtime is not affected much by the sample size thanks to the multi-threading capability of sPLINK's client package, and linearly/non-linearly increases with the number of SNPs/clients. The corresponding plots are available in Additional file 1: Fig. S3, S4, and S5.

\section{Discussion}

We first provide a general discussion on the privacy of the existing tools for collaborative GWAS including SPLINK. To be more accurate, we draw a distinction between the privacy-aware and privacy-preserving definitions [39]. In a privacy-aware approach, it is not required to share the private data with a third party. A privacy-aware approach is privacy-preserving if the approach offers a privacy guarantee that captures the privacy risk associated with individual samples in the dataset. Given that, meta-analysis, SMPC, HE, federated learning, and hybrid federated learning based on SMPC are privacy-aware because they do not share the raw data with a third party. In meta-analysis/federated learning, the summary statistics/model parameters of each cohort are shared with a third party. In SMPC-based hybrid federated learning, the aggregated (global) parameters are revealed to the server and cohorts. These approaches, including HE and SMPC, reveal the final model too. However, these methods are not privacy-preserving because none of them provides a privacy guarantee indicating to what extent the revealed information leaks the private data of a particular sample in the dataset. To our knowledge, differential privacy (DP) [40] and DP-based hybrid federated learning can offer such a guarantee at the cost of the utility of the model and are considered as privacy-preserving approaches.

While privacy-aware approaches do not offer a privacy guarantee, they might provide stronger/weaker privacy compared to each other based on the amount and nature of the information they share with third parties. For instance, HE-based methods provide stronger privacy because they only reveal the final model (results) while other privacyaware approaches disclose not only the final results but also other information such as summary statistics or local parameters. Similarly, sPLINK provides enhanced privacy in comparison with existing federated learning based tools such as GLORE. This is because GLORE discloses the local parameters of each cohort to the server, which is not revealed in $S P L I N K$.

sPLINK is a privacy-aware tool, assuming honest-but-curious server, compensator, and clients, which (I) follow the protocol as it is; for instance, the server always sends the global beta values resulted from the aggregation but not the beta values tampered with such as all zeros to the clients, and (II) do not collude with each other, e.g. the compensator never shares the individual noise values of the clients with the server and similarly, the server does not send the noisy local parameters to the compensator, but (III) they try to reconstruct the raw data using the model parameters. Additionally, (IV) there are at least 
three different cohorts participating in the study, and their client components as well as the server and compensator components are running in separate physical machines.

Given these assumptions, we discuss the privacy of the masking mechanism of sPLINK (inherited from $\mathrm{HyFed}$ ) for the supported association tests. To this end, we use the information theoretic criterion called mutual information between two random variables $X$ and $Y[30,41]$ :

$$
I(X, Y)=H(X)-H(X \mid Y)
$$

where $H(X)$ and $H(X \mid Y)$ indicate the entropy of $X$ and the conditional entropy of $X$ given $Y$, respectively. The mutual information measures (in bits) the decrease in uncertainty about $X$ having the knowledge of $Y$. In $s P L I N K$, the noisy local parameter $M_{L}^{\prime}$ is a secret share from the local parameter $M_{L}$ (the secret), and random variables $X$ and $Y$ indicate the distributions of $M_{L}$ and $M_{L}^{\prime}$, respectively.

The local parameter $M_{L}$ of a client is either a non-negative integer (e.g. sample count, allele count, or contingency table) or floating-point number (e.g. Hessian or covariance matrix) in the association tests. For non-negative integers, sPLINK capitalizes on additive secret sharing based on modular arithmetic over the finite field $\mathbb{Z}_{p}=\{0,1, p-1\}$, in which $p$ is a prime number [13]. For floating-point numbers, sPLINK employs real value secret sharing based on Gaussian (Normal) distribution [42, 43] (more details in "Methods" section).

For non-negative integers, noise $N_{L}$ is generated from a uniform distribution over $\mathbb{Z}_{p}$, and $M_{L}^{\prime}$ is the modular addition of $M_{L}$ and $N_{L}: M_{L}^{\prime}=\left(M_{L}+N_{L}\right) \bmod p$. For this scheme, it has been shown that the knowledge of $Y$ (noisy local parameter) provides no information about $X$ (local parameter), which means the mutual information between them is zero: $I(X, Y)=0[13,16]$. Notice that this is the case for any value of prime number $p$.

For floating-point numbers, noise $N_{L}$ is generated using Gaussian distribution with variance of $\sigma_{N}^{2}$. Assuming that the variance of $X$ is $\sigma_{M_{L}}^{2}$, the mutual information between $X$ and $Y$ is maximum if $Y$ follows the Gaussian distribution (variance $\sigma_{M_{L}}^{2}+\sigma_{N}^{2}$ ) [43]. Thus, the upper bound on the mutual information between $X$ and $Y$ is:

$$
I(X, Y)=\frac{1}{2} \log _{2}\left(1+\frac{\sigma_{M_{L}}^{2}}{\sigma_{N}^{2}}\right)
$$

That is, the amount of reduction in uncertainty about the local parameters having the knowledge of the noisy local parameters depends on the relative variance of the corresponding distributions. Therefore, using larger values for variance in the Gaussian random generator will provide lower information leakage. The value of mean for the Gaussian random generator does not remarkably impact the privacy and can be set to zero [43], which is the case for sPLINK. The default value of $\sigma_{N}^{2}$ is $10^{12}$ for sPLINK, which is large enough for typical GWAS, but it can be set to higher values if needed to ensure that $\frac{\sigma_{M_{L}}^{2}}{\sigma_{N}^{2}}$ remains small.

Notice that although sPLINK significantly enhances the privacy of data compared to existing federated learning tools by hiding the local parameters of clients from a third party, it does not eliminate the possibility of data reconstruction using the aggregated parameters or final results. For example, the $X^{T} X$ parameter (covariance matrix) in the linear regression algorithm can be exploited to determine the sex of the patients if the 
total number of samples across all cohorts is comparable to the number of the confounding factors. However, for a reliable GWAS study, the total sample size is considerably larger than the number of confounding factors, and therefore, the reconstruction of the cohorts' private data from the aggregated parameters can be difficult (but still possible) in practice. A similar argument is also applicable to meta-analysis approaches, which reveal the summary statistics of each cohort to a third party.

The value of prime number $p$ impacts the correctness of the masking mechanism. To ensure the correctness, overflow must not occur in $\sum_{i=1}^{i=K} N_{L_{i}}$ and $\sum_{i=1}^{i=K} M_{L_{i}}^{\prime}$ calculations, and $\sum_{i=1}^{i=K} M_{L_{i}}<p$. sPLINK uses the default value of $p=2^{54}-33$, which is the largest prime number than can fit in 54-bit integer. A higher value of $p$ can be employed to handle larger integer values but at the expense of a lower number of clients [30]. Likewise, too large values of variance $\sigma_{N}^{2}$ (e.g. $10^{30}$ ) can impact the precision of the results. With default values of $p$ and $\sigma_{N}^{2}$, however, our experiments indicate that there are no statistically significant differences between the results from sPLINK with and without the masking mechanism for all three association tests (the experimental setup of Fig. 7 is used in the experiments).

SPLINK currently supports chi-square and linear/logistic regression tests, but it can be extended to compute other useful statistics in GWAS such as minor allele frequency (MAF), Hardy-Weinberg equilibrium (HWE), and linkage disequilibrium (LD) between SNPs in a privacy-aware manner. The federated computation of the aforementioned statistics in SPLINK is expected to be straightforward because they are based on the allele frequencies, and SPLINK already calculates the minor and major allele counts in the Nonmissing count step of its computational workflow (the "Methods" section). Moreover, population stratification using the principal component analysis (PCA) will be addressed in the future version of SPLINK due to the complexity of the problem. sPLINK's implementation of the association tests is horizontally-federated, where the datasets have different samples but the same features (i.e. SNP and confounding factors). However, correcting for population structure using SPLINK requires a vertically-federated [44] PCA algorithm because the eigenvectors should be computed from the sample by sample covariance matrix, and therefore, the samples and features swap roles in the federated PCA (SNPs are considered as samples and patients as features) [45]. Vertical federated learning algorithms are still understudied, and they are considered more complicated than the horizontal algorithms.

Additionally, the federated PCA algorithm should be an iterative, randomized algorithm [46] so that it can handle large GWAS datasets with a practical amount of main memory. The iterative nature of the algorithm will present network and runtime challenges because it might need dozens or hundreds of iterations and exchange huge traffic over the network to converge to the final eigenvectors. From the privacy perspective, a recent study [45] demonstrates that even if we assume the federated PCA and linear regression algorithms individually provide perfect privacy, federated population stratification in GWAS, where the eigenvectors are used as the confounding factors in the association test, does not necessarily offer perfect privacy. Consequently, the server can reconstruct the SNP or binary confounding factor values in polynomial time. To tackle this issue, they suggested that the final eigenvectors should be computed at the clients and the model parameter values should be hidden from the server. The federated population stratification in sPLINK should be implemented taking into account those suggestions. 
We showed that sPLINK is robust against an important source of data heterogeneity, namely the heterogeneous distribution of the phenotype or confounding factor values across the distributed datasets of the cohorts. Population heterogeneity across the cohorts is another source of data heterogeneity in GWAS, which is commonly tackled by population stratification using the PCA algorithm. sPLINK currently does not address this kind of data heterogeneity but the future versions of the tool will support population stratification to this end.

\section{Conclusions}

We introduce sPLINK, a user-friendly, hybrid federated tool for GWAS. sPLINK enhances the privacy of the cohorts' data without sacrificing the accuracy of the test results. It supports multiple association tests including chi-square, linear regression, and logistic regression. SPLINK is consistent with PLINK in terms of the input data formats and results. We compare $S P L I N K$ to aggregated analysis with PLINK as well as meta-analysis with METAL, GWAMA, and PLINK. While SPLINK is robust against the heterogeneity of phenotype or confounding factor distributions across separate datasets, the statistical power of the meta-analysis tools is declined in imbalanced/heterogeneous scenarios. We argue that SPLINK is easier to use for collaborative GWAS compared to metaanalysis approaches thanks to its straightforward functional workflow. We also show that SPLINK achieves practical runtime, in order of minutes or hours, and acceptable network usage. SPLINK is an open-source tool and its source code is publicly available under the Apache License Version 2.0. sPLINK is a novel and robust alternative to meta-analysis, which performs collaborative GWAS in a privacy-aware manner. It has the potential to immensely impact the statistical genetics community by addressing current challenges in GWAS including cross-study heterogeneity and, thus, to replace meta-analysis as the gold standard for collaborative GWAS.

\section{Methods}

Federated learning $[14,15]$ is a type of distributed learning, where multiple cohorts collaboratively learn a joint (global) model under the orchestration of a central server [47]. The cohorts never share their private data with the server or the other cohorts. Instead, they extract local parameters from their data and send them to the server. The server aggregates the local parameters from all cohorts to compute the global model parameters (or global results), which in turn, are shared with all cohorts. While federated learning is privacy-aware, where the private data of the cohorts is not shared with the server, studies $[48,49]$ have shown that for some models such as deep neural networks, the raw data can be reconstructed from the parameters shared by the cohorts.

To improve the privacy of federated learning, privacy-enhancing technologies (PETs) such as DP, HE, or SMPC can be combined with federated learning to avoid revealing the original values of the local parameters to third parties including the server [50]. DP-based hybrid federated learning approaches can provide a privacy guarantee but their final results might be considerably impacted by the random noise employed for the perturbation of the model. HE-based aggregation methods can incur remarkable computational overhead because they require the cohorts to encrypt/decrypt the local/global model parameters and the server to perform the aggregation over the encrypted parameters. SMPC-based hybrid federated learning methods $[30,51]$ increase the network bandwidth 
usage but does not adversely affect the final results. HyFed is an open-source hybrid federated framework, which combines federated learning with additive secret sharingbased SMPC to enhance the privacy of the federated algorithms while preserving the utility (performance) of the global model. HyFed provides a generic API (application programming interface) to develop federated machine learning algorithms. It supports the federated mode of operation, where different components of the framework can be installed in separate physical machines and securely communicate with each other through the Internet.

sPLINK implements a hybrid federated approach using the HyFed API to enhance the privacy of data. sPLINK works with distributed GWAS data, where samples are individuals and features are SNPs and categorical or quantitative phenotypic variables. While the samples are different across the cohorts, the feature space is the same because sPLINK only considers SNPs and phenotypic variables that are common among all datasets (horizontal or sample-based federated learning)[44]. The client package of sPLINK is installed on the local machine of each cohort with access to the private data. The compensator is running in a separate machine. sPLINK's server and WebApp packages are installed on a central server.

In $s P L I N K$, the original values of the parameters computed from the private data in one cohort is not revealed to the server, compensator, or other cohorts, improving the privacy of the cohorts' data. SPLINK provides the chunking capability to handle large datasets containing millions of SNPs. The chunk size (configured by the coordinator) specifies how many SNPs should be processed in parallel. Larger chunk sizes allow for more parallelism, and therefore less running time in general but require more computational resources (e.g. CPU and main memory) from the local machines of the cohorts, the server, and compensator. sPLINK's client package is multi-threaded, where the number of cores is configurable by the participants. This makes the computation of the model parameters in the cohorts very fast, especially for large datasets. While we provide a readily usable web service running at exbio server (https://exbio.wzw.tum.de/splink) and online compensator at compbio server (https://compensator.compbio.sdu.dk), the server, WebApp, and compensator packages can, of course, be deployed on customized physical machines.

The functional workflow of SPLINK is comprised of the following steps:

1. Project creation: The coordinator creates the project (new study) through the Web interface. To this end, she/he first specifies the project name, association test name, chunk size, and the list of confounding features (only for regression tests), and then, generates a unique project token for each cohort.

2. Cohort invitation: The coordinator sends the project ID (automatically generated) and token to each participant (a human entity interacting with the client package in a cohort) through a secure channel such as email for inviting the cohorts to the project.

3. Cohort joining: The participants use their corresponding username, password, project ID, and token to join the project. After joining, they can view the general information of the project such as the coordinator, server/compensator name/URL, and etc. If they agree to proceed, they choose the dataset they want to employ in the study. To be consistent with PLINK, sPLINK supports .bed (value of SNPs), fam (sample IDs as well as sex and phenotype values), .bim (chromosome 
number, name, and base-pair distance of each SNP), .cov (value of confounding factors), and .pheno (phenotype values that should be used instead of those in .fam file) file formats as specified in the PLINK manual [52]. For linear regression, phenotype values must be quantitative while for logistic regression and chi-square, phenotype values have to be binary (control/case are encoded as $1 / 2$ ).

4. Federated computation: In $S P L I N K$, the association test results are computed by the client package (running on the local machines of cohorts), server package (running in the central server), and compensator (running in its own machine) in a federated manner. The computation is iterative and consists of six general steps:

(a) Get global parameters: All clients obtain the required global parameters $M_{G}$ from the server.

(b) Compute local parameters: Each client i computes the local parameters $M_{L_{i}}$ using the local data and global parameters.

(c) Mask local parameters: Each client $i$ generates random noise $N_{L_{i}}$ with the same shape as $M_{L_{i}}$, and masks $M_{L_{i}}$ with $N_{L_{i}}$ to obtain the noisy local parameters $M_{L_{i}}^{\prime}$.

(d) Share noisy local parameters and noise: Each client $i$ shares $M_{L_{i}}^{\prime}$ and $N_{L_{i}}$ with the server and compensator, respectively.

(e) Aggregate noise: The compensator computes the aggregated noise $N$ given the noise values from the clients and sends the aggregated noise $N$ to the server.

(f) Compute global parameters: The server calculates (unmasks) the global parameters given the noisy local parameters and the negative of the aggregated noise.

5. Result download: The final results are automatically downloaded for the cohorts but the coordinator needs to download them manually through the web interface. Similar to PLINK, SPLINK reports minor allele name $(A 1)$ and $p$-value $(P)$ for all three association tests, chi-square (CHISQ), odds ratio $(O R)$, minor allele frequency in cases $\left(F \_A\right)$, and minor allele frequency in controls $\left(F \_U\right)$ for chi-square test, and the number of non-missing samples (NMISS), beta (BETA), and t-statistic (STAT) for linear and logistic regression tests.

sPLINK inherits its masking mechanism from $\mathrm{HyFed}$, which masks the local parameters with non-negative integer and floating-point values in different ways. For a local parameter with a non-negative integer value, $s P L I N K$ considers a finite field $\mathbb{Z}_{p}=\{0,1, p-1\}(p$ is a prime number) [13], where each client $i$ generates a uniform random integer from $\mathbb{Z}_{p}$ as noise $N_{L_{i}}$ and masks its local parameter $M_{L_{i}}$ with $N_{L_{i}}$ by performing the modular addition over $\mathbb{Z}_{p}: M_{L_{i}}^{\prime}=\left(M_{L_{i}}+N_{L_{i}}\right) \bmod p$. Notice that $M_{L_{i}}, N_{L_{i}}, M_{L_{i}}^{\prime} \in \mathbb{Z}_{p}$. For $M_{L_{i}}$ with a floating-point value, each client $i$ generates noise $N_{L_{i}}$ using Gaussian random generator with zero-mean and variance $\sigma_{N}^{2}$, and masks $M_{L_{i}}$ with $N_{L_{i}}$ using the ordinary addition: $M_{L_{i}}^{\prime}=M_{L_{i}}+N_{L_{i}}$.

The compensator computes the aggregated noise $N$ by taking sum over the noise values of the clients using the modular or ordinary addition depending on the data type of the noise: if $N_{L_{i}}$ is non-negative integer, then $N=\left(\sum_{i=1}^{i=K} N_{L_{i}}\right) \quad \bmod p$; if $N_{L_{i}}$ is floatingpoint type, then $N=\sum_{i=1}^{i=K} N_{L_{i}}$. To calculate the global parameters with non-negative 
integer values, the server first computes the aggregated noisy parameter by taking sum over the noisy local parameters using the modular addition, and then subtracts the aggregated noise from the aggregated noisy parameter using the modular subtraction: $M_{G}=$ $\left(\left(\left(\sum_{i=1}^{i=K} M_{L_{i}}^{\prime}\right) \bmod p\right)-N\right) \bmod p$. For model parameters with floating-point values, the server adds up the noisy local parameters and the negative of the aggregated noise using the ordinary addition: $M_{G}=\sum_{i=1}^{i=K} M_{L_{i}}^{\prime}-N$.

The computational workflow of sPLINK involves seven steps common among all association tests as well as a couple of steps specific to each association test (Fig. 8). In the first three steps (i.e. Init, SNP name, and Allele name) as well as the sixth step (Minor allele), the clients only communicate with the server, where the name of the SNPs and alleles (which are not considered private) are directly shared with the server. In the remaining steps, the compensator is involved and clients mask the local parameters with noise to hide their original values from the server. The formulas associated with the steps indicate how the clients compute local parameters and how the server calculates the global parameters using the noisy local parameters of the clients and the aggregated noise from the compensator. In the following, we provide an overview of each step:

1. Init: Each client $i$ opens the files of the dataset selected by the participant to be employed in the study and creates its phenotype vector $\left(Y_{i}\right)$ and feature matrix $\left(X_{i}\right)$, which includes the value of SNPs and confounding factors. It is worth noting that there is a separate feature matrix for each SNP but the phenotype vector is the same for all SNPs. Assume a dataset containing three SNPs named SNP1, SNP2, and SNP3 and age and sex as confounding features. There will be three different feature matrices, one feature matrix per SNP. For instance, the feature matrix of SNP1 has three columns including SNP1, age, and sex values. Phenotype vector and feature matrix are the private data of the cohorts. They cannot be shared with the server, compensator, or the other cohorts. The aggregation process in the server just makes sure that all clients successfully initialized their data.

2. SNP name: Each client shares the SNP names with the server. In the aggregation process, the server computes the intersection of all SNP names. Only common SNPs are considered in the computation of the association test results.

3. Allele name: Each client sends the allele names (e.g. G,A) of each SNP to the server. In the aggregation process, the server ensures that all cohorts employ the same allele names for the SNPs. Notice that the clients sort the allele names to avoid revealing which one is minor or major allele.

4. Sample count: Each client $i$ calculates its local sample count $T_{i}$ (number of samples in its dataset including missing samples, which is the size of vector $\left.Y_{i}\right)$. The server computes the corresponding global sample count: $T=\left(\left(\left(\sum_{i=1}^{i=K} T_{i}^{\prime}\right)\right.\right.$ $\left.\bmod p)-N_{T}\right) \bmod p$, where $T_{i}^{\prime}$ is the noisy local sample count of client $i: T_{i}^{\prime}=$ $\left(T_{i}+N_{i}\right) \bmod p$ and $N_{T}$ is the aggregated noise from the compensator: $N_{T}=$ $\left(\sum_{i=1}^{i=K} N_{i}\right) \bmod p$.

5. Non-missing count: In this step, SNPs are split into chunks which can be processed in parallel. The chunking capability is provided to handle very large datasets containing millions of SNPs. The clients compute the non-missing sample count by filtering out the missing samples (value of -9 is considered as missing). Likewise, they calculate the local allele count by counting the number of alleles in 


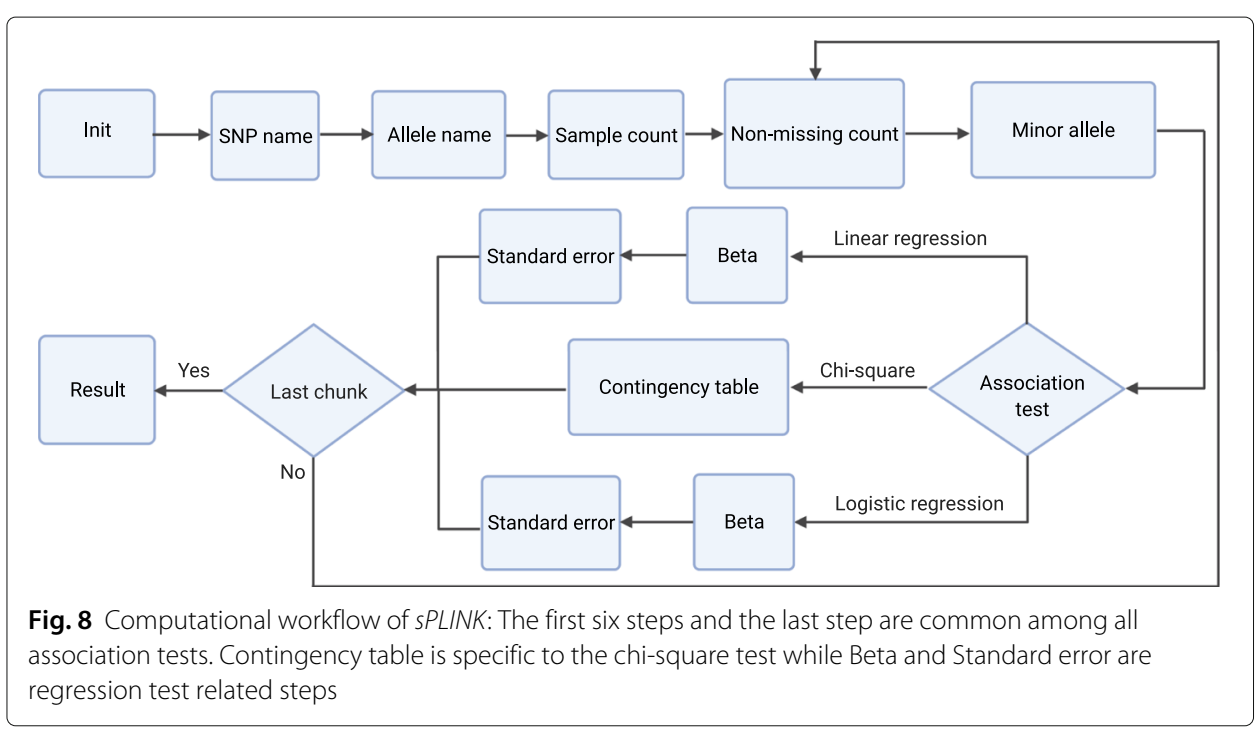

each SNP. In the aggregation process, the server computes the global non-missing sample count $(n)$ and allele count using the corresponding noisy parameters and the aggregated noise similar to the sample count step. Finally, the server determines the global minor allele based on the values of the global allele counts.

6. Minor allele: The clients compare their local minor allele with the global minor allele. If they are the same, they do nothing. Otherwise, they update the mapping of SNP values read from .bed file. Each SNP value can be 0,1 , 2, or 3 (missing value). These values are encoded based on the minor allele name. If the minor allele is changed, the value of the SNP needs to be swapped if it is 0 or 2. Thus, if a client's minor allele is different from global minor allele, it inverses the mapping of SNP values $(0 \rightarrow 2$ and $2 \rightarrow 0)$. The aggregation in the server makes sure that all clients successfully completed this step.

7. Association test specific steps: In the following, we elaborate on the steps specific to each association test. Regarding regression tests, sPLINK implements the federated versions of ordinary least squares linear regression and Newton-Raphson method based logistic regression.

Chi-square: The only test-specific step for the chi-square test is Contingency table, where each client $i$ computes its local contingency table containing minor allele frequency for cases $\left(t_{i}\right)$, minor allele frequency for controls $\left(r_{i}\right)$, major allele frequency for cases $\left(q_{i}\right)$, and major allele frequency for controls $\left(s_{i}\right)$. The server aggregates the noisy contingency tables from the clients $\left(t_{i}^{\prime}, r_{i}^{\prime}, q_{i}^{\prime}\right.$, and $s_{i}^{\prime}$ are the elements of the table) and the corresponding aggregated noise from the compensator $\left(N_{t}, N_{r}, N_{q}\right.$, and $N_{s}$ ) to compute the global (observed) contingency table (Table 3). It also calculates the expected contingency table based on the observed contingency table (Table 4).

Given the observed contingency table $(O)$ and the expected contingency table $(E)$, the server computes odds ratio (OR), $\chi^{2}$, and $p$-value $(P)$ as follows:

$$
\mathrm{OR}=\frac{t \times s}{q \times r}
$$


Table 3 Global (observed) contingency table

\begin{tabular}{|c|c|c|c|}
\hline & Minor allele & Major allele & Total \\
\hline Case & $t=\left(\left(\left(\sum_{i=1}^{i=k} t_{i}^{\prime}\right) \bmod p\right)-N_{t}\right) \bmod p$ & $q=\left(\left(\left(\sum_{i=1}^{i=K} q_{i}^{\prime}\right) \bmod p\right)-N_{q}\right) \bmod p$ & $t+q$ \\
\hline Control & $r=\left(\left(\left(\sum_{i=1}^{i=K} r_{i}^{\prime}\right) \bmod p\right)-N_{r}\right) \bmod p$ & $s=\left(\left(\left(\sum_{i=1}^{i=K} s_{i}^{\prime}\right) \bmod p\right)-N_{s}\right) \bmod p$ & $r+s$ \\
\hline Total & $t+r$ & $q+s$ & $2 n$ \\
\hline
\end{tabular}

$$
\begin{aligned}
& \chi^{2}=\sum \frac{(E-O)^{2}}{E} \\
& P=1-F_{t}\left(\chi^{2}, 1\right)
\end{aligned}
$$

where $F_{t}$ is the cumulative distribution function (CDF) of $\chi^{2}$ distribution (degree of freedom is 1 ).

Linear regression: Beta and Standard error are two steps specific to linear regression test. In the Beta step, each client $i$ computes $X_{i}^{T} X_{i}$ and $X_{i}^{T} Y_{i}$, where $X_{i}^{T}$ is the transpose of $X_{i}$. In the aggregation process, the server performs the following calculations ( $K$ is the number of clients):

$$
\begin{aligned}
& X^{T} X=\sum_{i=1}^{i=K}\left(X_{i}^{T} X_{i}\right)^{\prime}-N_{X^{T} X} \\
& X^{T} Y=\sum_{i=1}^{i=K}\left(X_{i}^{T} Y_{i}\right)^{\prime}-N_{X^{T} Y} \\
& \beta=\left(X^{T} X\right)^{-1}\left(X^{T} Y\right)
\end{aligned}
$$

where $\left(X_{i}^{T} X_{i}\right)^{\prime}$ and $\left(X_{i}^{T} Y_{i}\right)^{\prime}$ are the noisy local parameters from the clients, $N_{X^{T} X}$ and $N_{X^{T} Y}$ are the corresponding aggregated noise from the compensator, and () ${ }^{-1}$ indicates the inverse matrix.

In the Standard error step, each client $i$ calculates the local sum square error (SSE) $E_{i}$ by having the global $\beta$ vector.

$$
\begin{aligned}
& \hat{Y}_{i}=X_{i} \beta \\
& E_{i}=\sum\left(Y_{i}-\hat{Y}_{i}\right)^{2}
\end{aligned}
$$

and then the server calculates the global standard error vector (SE) as follows:

$$
\begin{aligned}
& E=\sum_{i=1}^{i=K} E_{i}^{\prime}-N_{E} \\
& \mathrm{VAR}=\left(\frac{E}{n-m-1}\right)\left(X^{T} X\right)^{-1} \\
& \mathrm{SE}=\sqrt{\operatorname{diag(\mathrm {VAR})}}
\end{aligned}
$$

Table 4 Expected contingency table

\begin{tabular}{llc}
\hline & Minor allele & Major allele \\
\hline Case & $\frac{(t+q) \times(t+r)}{2 n}$ & $\frac{(t+q) \times(q+s)}{2 n}$ \\
Control & $\frac{(r+s) \times(t+r)}{2 n}$ & $\frac{(r+s) \times(q+s)}{2 n}$ \\
\hline
\end{tabular}


where $E_{i}^{\prime}$ and $N_{E}$ are the noisy SSE values and the corresponding aggregated noise, respectively; $n$ is the global non-missing sample count, $m$ is the number of features ( $1+$ number of confounding factors), and diag is the main diagonal of the matrix. Given the standard error vector, the server computes the $T$ statistic $(T)$ and $p$-value $(P)$ as follows:

$$
\begin{aligned}
& T=\frac{\beta}{\mathrm{SE}} \\
& \mathrm{DF}=n-m-1 \\
& P=2 \times\left(1-F_{t}(|T|, \mathrm{DF})\right)
\end{aligned}
$$

in which $D F$ is degree of freedom and $F_{t}$ is the CDF of $\mathrm{T}$ distribution.

Logistic regression: Similar to linear regression, logistic regression has two specific steps: Beta and Standard error. However, the Beta step is iterative in logistic regression (maximum number of iterations is specified by the coordinator and its default value is 20). In each iteration, each client $i$ computes local gradient $\left(\nabla_{i}\right)$, Hessian matrix $\left(H_{i}\right)$ and $\log$-likelihood $\left(L_{i}\right)$ as follows:

$$
\begin{aligned}
\hat{Y}_{i} & =\frac{1}{1+e^{-X_{i} \beta}} \\
\nabla_{i} & =X_{i}^{T}\left(Y_{i}-\hat{Y}_{i}\right) \\
H_{i} & =\left(X_{i}^{T} \circ\left(\hat{Y}_{i} \circ\left(1-\hat{Y}_{i}\right)\right)^{T}\right) X_{i} \\
L_{i} & =\sum\left(Y_{i} \circ \log \hat{Y}_{i}+\left(1-Y_{i}\right) \circ \log \left(1-\hat{Y}_{i}\right)\right)
\end{aligned}
$$

where $\beta$ is the global beta vector from the previous iteration and $\circ$ indicates element-wise multiplication.

The server aggregates the noisy local gradients $\left(\nabla_{i}^{\prime}\right)$, Hessian matrices $\left(H_{i}^{\prime}\right)$ and $\log$-likelihood values $\left(L_{i}^{\prime}\right)$ from $K$ clients and the associated aggregated noise values $N_{\nabla}, N_{H}, N_{L}$ as follows:

$$
\begin{aligned}
& \nabla=\sum_{i=1}^{i=K} \nabla_{i}^{\prime}-N_{\nabla} \\
& H=\sum_{i=1}^{i=K} H_{i}^{\prime}-N_{H} \\
& L=\sum_{i=1}^{i=K} L_{i}^{\prime}-N_{L}
\end{aligned}
$$

Then, it updates the $\beta$ values accordingly:

$$
\beta_{\text {new }}=\beta_{\text {old }}+H^{-1} \nabla
$$

where $\beta_{\text {old }}$ is the $\beta$ value from the previous iteration. The server also compares the newly computed log-likelihood value $(\mathrm{L})$ with the one from previous iteration $\left(L_{\text {old }}\right)$. If their difference is less than a pre-specified threshold, $\beta$ values converged, and therefore, it stops updating beta. 
In the Standard error step, the server shares the global $\beta$ values with the clients. Each client $i$ computes its local Hessian matrix $\left(H_{i}\right)$ using the global $\beta$. The server gets the noisy local Hessian matrices from $K$ clients and the aggregated noise from the compensator and applies the following formula to obtain the global standard error vector (SE):

$$
\mathrm{SE}=\sqrt{\operatorname{diag}\left(\left(\sum_{i=1}^{i=K} H_{i}^{\prime}-N_{H}\right)^{-1}\right)}
$$

Having standard error values, the server calculates $T$ statistics and $p$-value $(P)$ as follows:

$$
\begin{aligned}
& T=\frac{\beta}{\mathrm{SE}} \\
& P=1-F_{t}\left(|T|^{2}, 1\right)
\end{aligned}
$$

where $F_{t}$ is CDF of $\chi^{2}$ distribution (degree of freedom is 1 ).

8. Result: The computation of association test results have been completed for all chunks and the results are shared with all cohorts.

The client and server components of sPLINK has been written using the Python API of the HyFed framework [53]. The WebApp component has been implemented using Angular and HTML/CSS. sPLINK employs the algorithm-agnostic compensator of the HyFed framework. The pandas package [54] is used in the client component to open the dataset files while NumPy [55] is leveraged to pre-process the data and to compute the local parameters. In the server component, the NumPy and SciPy [56] packages are used for aggregation and computing $p$-values.

\section{Supplementary Information}

The online version contains supplementary material available at https://doi.org/10.1186/s13059-021-02562-1.

\footnotetext{
Additional file 1: Experimental details. Table S1. The SHIP case study. Table S2. The COPDGene case study. Table S3. The FinnGen case study. Supplementary results. Figure S1. The significant SNPs overlapped between sPLINK and PLINK for the SHIP case study considering Bonferroni significance threshold. Figure S2. The Spearman rank correlation coefficient between the $p$-values from each tool and the aggregated analysis for the COPDGene and FinnGen case studies. Figure S3. Runtime and network bandwidth usage of sPLINK with varying number of SNPS. Figure S4. Runtime and network bandwidth usage of sPLINK with varying number of samples. Figure S5. Runtime and network bandwidth usage of sPLINK with varying number of clients. Experimental setup. Table S4. The system specification of the physical machines and laptops used to measure the runtime and network bandwidth usage of sPLINK. Table S5. The experimental setup used for measuring the runtime and network bandwidth usage of sPLINK.

Additional file 2: Review history.
}

\section{Acknowledgements}

We would like to thank Anne Hartebrodt and Richard Röttger for providing and setting up the workstation for the compensator component at the University of Southern Denmark. SHIP is part of the Community Medicine Research net of the University of Greifswald, Germany (https://www.community-medicine.de), which is funded by the Federal Ministry of Education and Research (grants no. 01ZZ9603, 01ZZ0103, and 01ZZ0403), the Siemens AG, the Ministry of Cultural Affairs as well as the Social Ministry of the Federal State of Mecklenburg-West Pomerania, and the network 'Greifswald Approach to Individualized Medicine (GANI_MED)' funded by the Federal Ministry of Education and Research (grant 03IS2061A). ExomeChip data have been supported by the Federal Ministry of Education and Research (grant no. 03Z1CN22) and the Federal State of Mecklenburg-West Pomerania. Patients and control subjects in FinnGen provided informed consent for biobank research, based on the Finnish Biobank Act. Alternatively, older research cohorts, collected prior the start of FinnGen (in August 2017), were collected based on study-specific consents and later transferred to the Finnish biobanks after approval by Fimea, the National Supervisory Authority for Welfare and Health. Recruitment protocols followed the biobank protocols approved by Fimea. The Coordinating Ethics Committee of the Hospital District of Helsinki and Uusimaa (HUS) approved the FinnGen study protocol Nr HUS/990/2017. The FinnGen project is approved by Finnish Institute for Health and Welfare (THL), approval number THL/2031/6.02.00/2017, amendments THL/1101/5.05.00/2017, THL/341/6.02.00/2018, THL/2222/6.02.00/2018, THL/283/6.02.00/2019 Digital and population 
data service agency VRK43431/2017-3, VRK/6909/2018-3, the Social Insurance Institution (KELA) KELA 58/522/2017, KELA 131/522/2018 and Statistics Finland TK-53-1041-17. The Biobank Access Decisions for FinnGen samples and data utilized in FinnGen Data Freeze 3 include: THL Biobank BB2017_55, BB2017_111, BB2018_19, BB_2018_34, BB_2018_67, BB2018_71, Red Cross Blood Service Biobank 7.12.2017, Helsinki Biobank HUS/359/2017, Auria Biobank AB17-5154, Biobank Borealis of Northern Finland_2017_1013, Biobank of Eastern Finland 1186/2018, Finnish Clinical Biobank Tampere MH0004, Central Finland Biobank 1-2017. The FinnGen project is funded by two grants from Business Finland (HUS 4685/31/2016 and UH 4386/31/2016) and eleven industry partners (AbbVie Inc, AstraZeneca UK Ltd, Biogen MA Inc, Celgene Corporation, Celgene International II Sàrl, Genentech Inc, Merck Sharp \& Dohme Corp, Pfizer Inc.,

GlaxoSmithKline, Sanofi, Maze Therapeutics Inc., Janssen Biotech Inc). Following biobanks are acknowledged for collecting the FinnGen project samples: Auria Biobank (https://www.auria.fi/biopankki), THL Biobank (https://www.thl.fi/ biobank), Helsinki Biobank (https://www.helsinginbiopankki.fi), Biobank Borealis of Northern Finland (https://www.ppshp fi/Tutkimus-ja-opetus/Biopankki/Pages/Biobank-Borealis-briefly-in-English.aspx), Finnish Clinical Biobank Tampere (https://www.tays.fi/en-US/Research_and_development/Finnish_Clinical_Biobank_Tampere), Biobank of Eastern Finland (https://www.ita-suomenbiopankki.fi/en), Central Finland Biobank (https://www.ksshp.fi/fi-Fl/Potilaalle/Biopankki), Finnish Red Cross Blood Service Biobank (https://www.veripalvelu.fi/verenluovutus/biopankkitoiminta) and Terveystalo Biobank (https://www.terveystalo.com/fi/Yritystietoa/Terveystalo-Biopankki/Biopankki/). All Finnish Biobanks are members of BBMRI.fi infrastructure (https://www.bbmri.fi). Figures 2 and 8 were created with BioRender.com.

\section{Peer review information}

Barbara Cheifet was the primary editor of this article and managed its editorial process and peer review in collaboration with the rest of the editorial team.

\section{Review history}

The review history is available as Additional file 2.

\section{Authors' contributions}

R.N., R.T., T.F., T.K., and J.B. conceived and designed the study. R.N. and R.T. developed the federated algorithms. R.N., R.T., and J.M. implemented the client and server components. J.M., R.N., R.T., T.F., and J.S. implemented the WebApp component. T.K. and R.N. performed the aggregated and federated association tests on the SHIP dataset. R.N., T.F., T.K., M.L., J.B., and S.W. conducted the meta-analysis on the COPDGene case study. R.N. and E.P. performed the meta-analysis on the FinnGen dataset. R.N., J.S., J.M., and R.T. conducted the performance measurements. R.N. and R.T. prepared the original draft. G.K. and D.R. provided critical feedback on the design and implementation of the tool from the privacy perspective. M.L., T.K., N.K.W., D.H., U.V., and J.B. helped with the manuscript revising. T.K., J.B., and M.L. assisted in the improvement of the tool. The authors read and approved the final manuscript.

\section{Funding}

This project has received funding from the European Union's Horizon 2020 research and innovation programme under grant agreement No 826078. This reflects only the author's view and the European Commission is not responsible for any use that may be made of the information it contains. This work was supported by the BMBF-funded de.NBI Cloud within the German Network for Bioinformatics Infrastructure (de.NBI) (031A537B, 031A533A, 031A538A, 031A533B, 031A535A, 031A537C, 031A534A, 031A532B). Open Access funding enabled and organized by Projekt DEAL.

\section{Availability of data and materials}

The SHIP dataset [33] is accessible to researchers after completing a web-based request form at http://ship.communitymedicine.de and approval. The COPDGene dataset [35] is publicly available (dbGaP accession number phs000179.v1.p1). The FinnGen dataset [36] is available for researchers by requesting access to the FinnGen Sandbox environment, and after completing Sandbox training on how to deal with personal data, and passing an exam about data security (https:// www.finngen.fi/en). The sPLINK tool is available online at https://exbio.wzw.tum.de/splink. The source code of sPLINK is publicly available at GitHub (https://github.com/tum-aimed/splink) and Zenodo (DOI: 10.5281/zenodo.5735472) [57] under the Apache License Version 2.0.

\section{Declarations}

\section{Ethics approval and consent to participate}

Not applicable.

\section{Competing interests}

The authors declare that they have no competing interests.

\section{Author details}

${ }^{1}$ Al in Medicine and Healthcare, Technical University of Munich, Munich, Germany. ${ }^{2}$ Klinikum rechts der Isar, Munich, Germany. ${ }^{3}$ Chair of Computational Systems Biology, University of Hamburg, Hamburg, Germany. ${ }^{4}$ Department of Mathematics and Computer Science, University of Southern Denmark, Odense, Denmark. ${ }^{5}$ Chair of Experimental Bioinformatics, TUM School of Life Sciences, Technical University of Munich, Munich, Germany. ${ }^{6}$ Department of Functional Genomics, University Medicine Greifswald, Greifswald, Germany. ${ }^{7}$ Institute for Molecular Medicine Finland (FIMM), Helsinki Institute of Life Science (HiLIFE), University of Helsinki, Helsinki, Finland. ${ }^{8}$ Applied Tumor Genomics Research Program, Research Programs Unit, Faculty of Medicine, University of Helsinki, Helsinki, Finland. ${ }^{9}$ Department of Mathematics and Computer Science, University of Marburg, Marburg, Germany. ${ }^{10}$ Division Data Science in Biomedicine, Peter L. Reichertz Institute for Medical Informatics of TU Braunschweig and Hannover Medical School, Brunswick, Germany. ${ }^{11}$ Braunschweig Integrated Centre of Systems Biology (BRICS), Brunswick, Germany. ${ }^{12}$ Biomedical Image Analysis Group, Imperial College London, London, UK. ${ }^{13}$ OpenMined, Oxford, UK. 
Received: 25 November 2020 Accepted: 2 December 2021

Published online: 24 January 2022

\section{References}

1. Fareed M, Afzal M. Single nucleotide polymorphism in genome-wide association of human population: A tool for broad spectrum service. Egypt J Med Human Genet. 2013;14(2):123-34.

2. Visscher PM, Wray NR, Zhang Q, Sklar P, McCarthy MI, Brown MA, Yang J. 10 years of gwas discovery: biology, function, and translation. Am J Hum Genet. 2017;101(1):5-22.

3. Visscher PM, Brown MA, McCarthy MI, Yang J. Five years of gwas discovery. Am J Hum Genet. 2012;90(1):7-24.

4. De R, Bush W, Moore J. Bioinformatics challenges in genome-wide association studies (gwas). Methods Mol Biol (Clifton, NJ). 2014;1168:63-81.

5. Purcell S, Neale B, Todd-Brown K, Thomas L, Ferreira MA, Bender D, Maller J, Sklar P, De Bakker PI, Daly MJ, et al. Plink: a tool set for whole-genome association and population-based linkage analyses. Am J Hum Genet. 2007;81(3): 559-75.

6. Evangelou $\mathrm{E}$, loannidis JP. Meta-analysis methods for genome-wide association studies and beyond. Nat Rev Genet. 2013;14(6):379-89.

7. Willer CJ, Li Y, Abecasis GR. Metal: fast and efficient meta-analysis of genomewide association scans. Bioinformatics. 2010;26(17):2190-1.

8. Mägi R, Morris AP. Gwama: software for genome-wide association meta-analysis. BMC Bioinformatics. 2010;11(1):288

9. Lunetta KL. Methods for meta-analysis of genetic data. Curr Protoc Human Genet. 2013;77(1):1-24.

10. Cantor RM, Lange K, Sinsheimer JS. Prioritizing gwas results: a review of statistical methods and recommendations for their application. Am J Hum Genet. 2010;86(1):6-22.

11. de Vlaming R, Okbay A, Rietveld CA, Johannesson M, Magnusson PK, Uitterlinden AG, van Rooij FJ, Hofman A, Groenen PJ, Thurik AR, et al. Meta-gwas accuracy and power (metagap) calculator shows that hiding heritability is partially due to imperfect genetic correlations across studies. PLoS Genet. 2017;13(1):e1006495.

12. Gentry C. Fully homomorphic encryption using ideal lattices. In: Proceedings of the Forty-first Annual ACM Symposium on Theory of Computing; 2009. p. 169-78.

13. Cramer R, Damgård IB, Nielsen JB. Secure Multiparty Computation and Secret Sharing. Cambridge: Cambridge University Press; 2015.

14. McMahan B, Moore E, Ramage D, Hampson S, y Arcas BA. Communication-efficient learning of deep networks from decentralized data. In: Artificial Intelligence and Statistics. Fort Lauderdale: PMLR; 2017. p. 1273-82.

15. Konečnỳ J, McMahan HB, Yu FX, Richtárik P, Suresh AT, Bacon D. Federated learning: Strategies for improving communication efficiency. arXiv preprint arXiv:1610.05492. 2016. https://arxiv.org/abs/1610.05492.

16. Shamir A. How to share a secret. Commun ACM. 1979;22(11):612-3.

17. Kamm L, Bogdanov D, Laur S, Vilo J. A new way to protect privacy in large-scale genome-wide association studies. Bioinformatics. 2013;29(7):886-93.

18. Cho H, Wu DJ, Berger B. Secure genome-wide association analysis using multiparty computation. Nat Biotechnol. 2018;36(6):547-51.

19. Shi H, Jiang C, Dai W, Jiang $X$, Tang Y, Ohno-Machado L, Wang S. Secure multi-party computation grid logistic regression (smac-glore). BMC Med Inf Dec Making. 2016;16(3):89.

20. Constable SD, Tang Y, Wang S, Jiang X, Chapin S. Privacy-preserving gwas analysis on federated genomic datasets. BMC Med Inf Dec Making. 2015;15:1-9.

21. Alexandru AB, Pappas GJ. Secure multi-party computation for cloud-based control. In: Privacy in Dynamical Systems. Singapore: Springer; 2020. p. 179-207.

22. Lu W-J, Yamada Y, Sakuma J. Privacy-preserving genome-wide association studies on cloud environment using fully homomorphic encryption. BMC Med Inf Dec Making. 2015;15:1-8.

23. Morshed T, Alhadidi D, Mohammed N. Parallel linear regression on encrypted data. In: 2018 16th Annual Conference on Privacy, Security and Trust (PST). Los Alamitos: IEEE Computer Society; 2018. p. 1-5.

24. Kim $M$, Song $Y$, Wang S, Xia $Y$, Jiang $X$, et al. Secure logistic regression based on homomorphic encryption: Design and evaluation. JMIR Med Inf. 2018;6(2):8805.

25. Sadat MN, Al Aziz MM, Mohammed N, Chen F, Jiang X, Wang S. Safety: secure gwas in federated environment through a hybrid solution. IEEE/ACM Trans Comput Biol Bioinforma. 2018;16(1):93-102.

26. Chialva D, Dooms A. Conditionals in homomorphic encryption and machine learning applications. arXiv preprint arXiv:1810.12380. 2018. https://arxiv.org/abs/1810.12380.

27. Wang S, Jiang X, Wu Y, Cui L, Cheng S, Ohno-Machado L. Expectation propagation logistic regression (explorer): distributed privacy-preserving online model learning. J Biomed Inf. 2013;46(3):480-96.

28. Wu Y, Jiang X, Kim J, Ohno-Machado L. Grid binary logistic regression (glore): building shared models without sharing data. J Am Med Inf Assoc. 2012;19(5):758-64.

29. Jiang W, Li P, Wang S, Wu Y, Xue M, Ohno-Machado L, Jiang X. Webglore: a web service for grid logistic regression. Bioinformatics. 2013;29(24):3238-40.

30. Nasirigerdeh R, Torkzadehmahani R, Matschinske J, Baumbach J, Rueckert D, Kaissis G. HyFed: A Hybrid Federated Framework for Privacy-preserving Machine Learning. arXiv preprint arXiv:2105.10545. 2021. https://arxiv.org/abs/ 2105.10545.

31. Hastie T, Tibshirani R, Friedman J. The Elements of Statistical Learning. Cambridge: Springer; 2009.

32. McHugh ML. The chi-square test of independence. Biochemia Med Biochemia Med. 2013;23(2):143-9.

33. Völzke H, Alte D, Schmidt CO, Radke D, Lorbeer R, Friedrich N, Aumann N, Lau K, Piontek M, Born G, et al. Cohort profile: the study of health in pomerania. Int J Epidemiol. 2011;40(2):294-307.

34. Weiss FU, Schurmann C, Guenther A, Ernst F, Teumer A, Mayerle J, Simon P, Völzke H, Radke D, Greinacher A, et al. Fucosyltransferase 2 (fut2) non-secretor status and blood group b are associated with elevated serum lipase activity in asymptomatic subjects, and an increased risk for chronic pancreatitis: a genetic association study. Gut. 2015;64(4):646-56 
35. COPDGene. http://www.copdgene.org/. Accessed 30 Nov 2021.

36. FinnGen Documentation of $R 3$ release. https://r3.finngen.fi/about. Accessed 30 Nov 2021.

37. Pillai SG, Ge D, Zhu G, Kong X, Shianna KV, Need AC, Feng S, Hersh CP, Bakke P, Gulsvik A, et al. A genome-wide association study in chronic obstructive pulmonary disease (copd): identification of two major susceptibility loci. PLoS Genet. 2009;5(3):e1000421.

38. Pei $Y-F$, Tian $Q$, Zhang L, Deng H-W. Exploring the major sources and extent of heterogeneity in a genome-wide association meta-analysis. Ann Hum Biol. 2016;80(2):113-22.

39. Lyu L, Yu H, Yang Q. Threats to federated learning: A survey. arXiv preprint arXiv:2003.02133. 2020. https://arxiv.org/ abs/2003.02133.

40. Dwork C. Differential privacy. In: International Colloquium on Automata, Languages, and Programming. Berlin: Springer; 2006. p. 1-12.

41. Cover TM. Elements of Information Theory. New York: John Wiley \& Sons; 1999.

42. Dibert A, Csirmaz L. Infinite secret sharing-examples. J Math Cryptol. 2014;8(2):141-68.

43. Tjell K, Wisniewski R. Privacy in Distributed Computations based on Real Number Secret Sharing. arXiv preprint arXiv:2107.00911. 2021. https://arxiv.org/abs/2107.00911.

44. Yang Q, Liu Y, Chen T, Tong Y. Federated machine learning: Concept and applications. ACM Trans Intell Syst Technol (TIST). 2019;10(2):1-19.

45. Nasirigerdeh R, Torkzadehmahani R, Baumbach J, Blumenthal DB. On the privacy of federated pipelines. In: Proceedings of the 44th International ACM SIGIR Conference on Research and Development in Information Retrieval (SIGIR '21). New York: Association for Computing Machinery; 2021.

46. Galinsky KJ, Bhatia G, Loh P-R, Georgiev S, Mukherjee S, Patterson NJ, Price AL. Fast principal-component analysis reveals convergent evolution of adh $1 \mathrm{~b}$ in europe and east asia. Am J Hum Genet. 2016;98(3):456-472.

47. Kairouz P, McMahan HB, Avent B, Bellet A, Bennis M, Bhagoji AN, Bonawitz K, Charles Z, Cormode G, Cummings R, et al. Advances and open problems in federated learning. arXiv preprint arXiv:1912.04977. 2019. https://arxiv.org/ $\mathrm{abs} / 1912.04977$.

48. Zhu L, Han S. Deep leakage from gradients. In: Federated Learning. Cham: Springer; 2020. p. 17-31.

49. Melis $L$, Song C, De Cristofaro E, Shmatikov V. Exploiting unintended feature leakage in collaborative learning. In: 2019 IEEE Symposium on Security and Privacy (SP). Manhattan: IEEE; 2019. p. 691-706.

50. Torkzadehmahani R, Nasirigerdeh R, Blumenthal DB, Kacprowski T, List M, Matschinske J, Späth J, Wenke NK, Bihari B, Frisch T, et al. Privacy-preserving Artificial Intelligence Techniques in Biomedicine. arXiv preprint arXiv:2007.11621. 2020. https://arxiv.org/abs/2007.11621.

51. Bonawitz K, Ivanov V, Kreuter B, Marcedone A, McMahan HB, Patel S, Ramage D, Segal A, Seth K. Practical secure aggregation for privacy-preserving machine learning. In: Proceedings of the 2017 ACM SIGSAC Conference on Computer and Communications Security. New York: Association for Computing Machinery; 2017. p. 1175-91.

52. PLINK data formats. http://zzz.bwh.harvard.edu/plink/data.shtml. Accessed 30 Nov 2021.

53. HyFed API. https://github.com/tum-aimed/hyfed. Accessed 30 Nov 2021.

54. pandas: Python Data Analysis Library. https://pandas.pydata.org/. Accessed 30 Nov 2021.

55. Harris CR, Millman KJ, van der Walt SJ, Gommers R, Virtanen P, Cournapeau D, Wieser E, Taylor J, Berg S, Smith NJ, Kern R, Picus M, Hoyer S, van Kerkwijk MH, Brett M, Haldane A, del Río JF, Wiebe M, Peterson P, Gérard-Marchant P, Sheppard K, Reddy T, Weckesser W, Abbasi H, Gohlke C, Oliphant TE. Array programming with NumPy. Nature. 2020;585(7825):357-62.

56. Virtanen P, Gommers R, Oliphant TE, Haberland M, Reddy T, Cournapeau D, Burovski E, Peterson P, Weckesser W, Bright J, van der Walt SJ, Brett M, Wilson J, Millman KJ, Mayorov N, Nelson ARJ, Jones E, Kern R, Larson E, Carey CJ, Polat I, Feng Y, Moore EW, VanderPlas J, Laxalde D, Perktold J, Cimrman R, Henriksen I, Quintero EA, Harris CR, Archibald AM, Ribeiro AH, Pedregosa F, van Mulbregt P, SciPy 1.0 Contributors. SciPy 1.0: Fundamental Algorithms for Scientific Computing in Python. Nat Methods. 2020;17:261-272.

57. Nasirigerdeh R, Torkzadehmahani R, Matschinske J, Frisch T, List M, Späth J, Weiss S, Völker U, Pitkänen E, Heider D, Wenke NK, Kaissis G, Rueckert D, Kacprowski T, Baumbach J. splink: a hybrid federated tool as a robust alternative to meta-analysis in genome-wide association studies. Zenodo. 2021. https://doi.org/10.5281/zenodo.5735472.

\section{Publisher's Note}

Springer Nature remains neutral with regard to jurisdictional claims in published maps and institutional affiliations.

Ready to submit your research? Choose BMC and benefit from:
- fast, convenient online submission
- thorough peer review by experienced researchers in your field
- rapid publication on acceptance
- support for research data, including large and complex data types
- gold Open Access which fosters wider collaboration and increased citations
- maximum visibility for your research: over 100M website views per year
At BMC, research is always in progress.
Learn more biomedcentral.com/submissions

